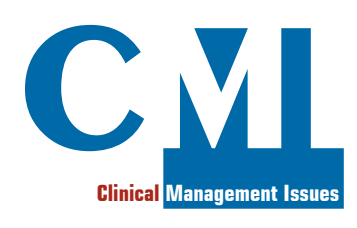

\title{
Un caso atipico di malattia da graffio di gatto
}

\begin{abstract}
A 53-year-old man arrives in emergency room for fever, asthenia and weight loss. Few days before the visit, he was treated with amoxicillin/clavulanic acid for a hard and not much sore submandibular swelling, with no result. Both ultrasound and CT showed the presence of colliquative multiple nodes, as well as multiple areas of hypoechoic nodules in the liver and spleen, granulomas related to pathognomonics of the hepatosplenic form of disease. In spite of a silent medical history and the lack of exposure to animal or insects, he resulted positive to IgM anti-Bartonella, and the diagnosis of cat scratch disease was confirmed by biopsy. We decided to treat with an association of ertapenem $1 \mathrm{~g} /$ day and teicoplanin $600 \mathrm{mg} /$ day for 3 weeks, and thus we obtained the recovery. The case report is of particular interest because of the lack of guidelines, the unusual manifestation of the disease and the singular associations of drugs administered.
\end{abstract}

Keywords: cat scratch disease, multiple adenopathy, unknown fever

An unusual case of cat scratch disease

CMI 2011; 5(Suppl 2):35-40

\section{CASO CLINICO}

Il signor $\mathrm{CP}$ giungeva alla nostra osservazione in Pronto Soccorso lo scorso autunno per febbre persistente da una settimana.

Si trattava di un impiegato cinquantatreenne, residente sulla collina torinese, che aveva da sempre goduto di ottima salute: infatti l'anamnesi patologica era praticamente muta a parte una pregressa reazione allergica ai chinolonici. In particolare, non emergevano fattori di rischio cardiovascolare né abitudini voluttuarie, e lo stile di vita prevedeva un'alimentazione varia e una modica attività fisica quotidiana.

La storia clinica del nostro paziente iniziava nei primi giorni di settembre, con la comparsa di insolita astenia e frequente febbricola prevalentemente serotina con puntate massime a $37,5^{\circ} \mathrm{C}$. Veniva inoltre riferita una concomitante e cospicua riduzione dell'apporto alimentare con calo pon- derale di $5 \mathrm{~kg}$. Circa 10 giorni prima della visita in ospedale era comparsa una tumefazione sottomandibolare destra di dimensioni ingravescenti fino a $3 \mathrm{~cm}$ di diametro, dura, ipomobile e poco dolente. L'ecografia del collo eseguita ambulatorialmente attribuiva la lesione a pacchetto linfonodale di aspetto reattivo, senza immagini riferibili a scialolitiasi. Su indicazione del medico di famiglia, il paziente aveva quindi avviato un trattamento antibiotico empirico con 2 $\mathrm{g} /$ die di amoxicillina/acido clavulanico per os, senza ottenere però alcun miglioramento clinico. L'andamento della febbre era da una settimana di tipo intermittente, con puntate fino a $40^{\circ} \mathrm{C}$ precedute da brivido scuotente e discretamente responsiva al paracetamolo.

Alla visita di ingresso il paziente era febbrile (temperatura $=38^{\circ} \mathrm{C}$ ), intensamente astenico ma vigile e orientato. La cute era calda e ben perfusa, integra in tutte le sedi esplorate. I valori di pressione arteriosa
${ }^{1}$ Medicina interna e DEA - Ospedale Santa Croce, Moncalieri 


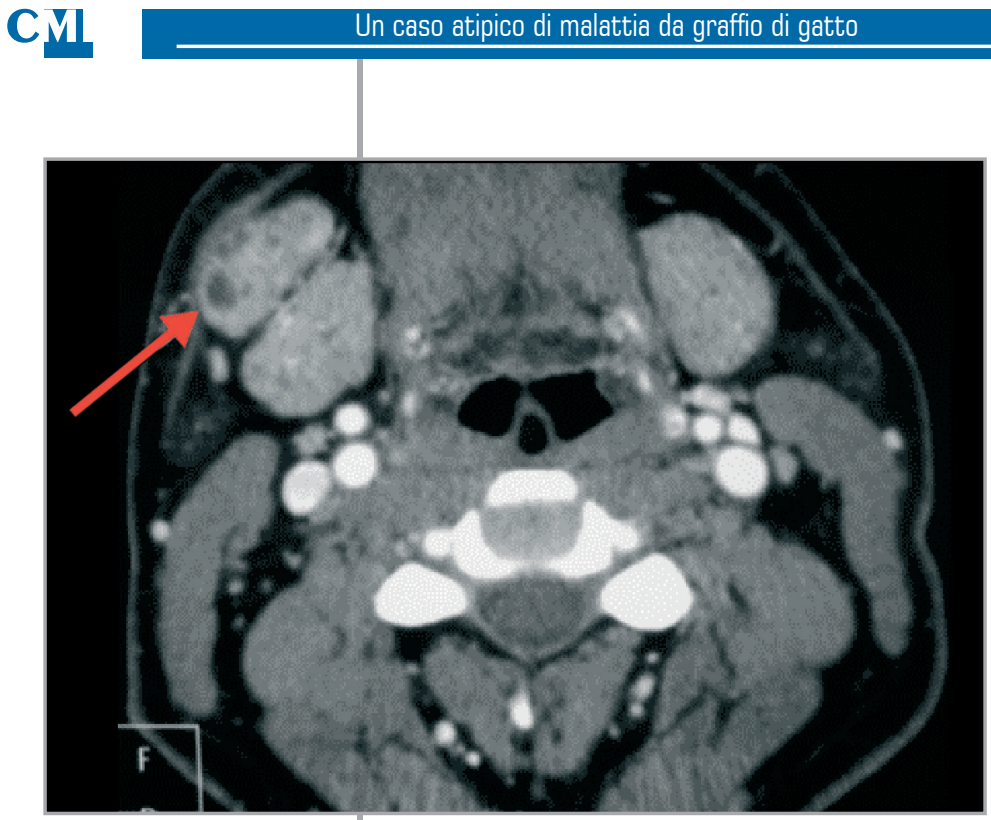

\section{Figura 1}

TC del collo con mdc. Si possono notare multiple aree colliquate (freccia) all'interno del pacchetto linfonodale, responsabili della tumefazione sottomandibolare

\section{Figura 2}

$T C$ torace e addome da cui si nota la presenza di multiple aree nodulari ipodense (frecce) nel fegato e nella milza erano $=130 / 95 \mathrm{mmHg}$ in clinostatismo e ortostatismo, la frequenza cardiaca $=100$ $\mathrm{bpm}$, la saturazione $=99 \%$ in aria ambiente, la frequenza respiratoria $=18$ atti al minuto. L'esame obiettivo cardiaco evidenziava toni ritmici e validi, con pause apparentemente libere; all'auscultazione del torace si apprezzavano normofonesi plessica e murmure diffuso senza rumori aggiunti. Alla palpazione l'addome era piano, trattabile, non dolorabile, con peristalsi valida; il fegato era nei limiti mentre era palpabile il polo splenico inferiore. Non erano visibili edemi, l'obiettività neurologica era del tutto nella norma. Anche il cavo orale era privo di lesioni, con faringe lievemente iperemica. Alla palpazione del collo si apprezzavano a sinistra multipli linfonodi laterocervicali e sottomandibolari mobili, di dimensioni inferiori a $1 \mathrm{~cm}$, mentre a destra era facilmente palpabile la tumefazione di circa $3 \mathrm{~cm}$, di consistenza duro-elastica e apparentemente adesa ai piani profondi, priva di segni di flogosi circostanti.

I primi esami ematochimici evidenziavano leucocitosi neutrofila con elevazione degli indici di flogosi acuta $(\mathrm{WBC}=12.000 / \mathrm{ml}$;
$\mathrm{PCR}=12 \mathrm{mg} / \mathrm{dl} ;$ fibrinogeno $=800 \mathrm{mg} / \mathrm{dl})$ e di LDH (600 UI/1). Si notava inoltre un lieve rialzo delle transaminasi $(\mathrm{AST}=53$ $\mathrm{UI} / 1 ; \mathrm{ALT}=111 \mathrm{UI} / 1)$. La funzione renale era conservata, così come i restanti parametri emocoagulativi e l'assetto degli elettroliti. Il test rapido della mononucleosi risultava negativo, l'Rx del torace era del tutto nella norma e allo striscio ematico periferico non veniva osservato nulla di rilevante.

Si decideva quindi di avviare un secondo ciclo antibiotico empirico, previa raccolta di campioni per emocoltura e urocoltura (il cui esito sarà negativo), con claritromicina ev $500 \mathrm{mg} / \mathrm{bid}$.

Le ulteriori indagini sierologiche effettuate durante il ricovero in Medicina Interna dimostravano iperferritinemia $(600 \mathrm{mg} / \mathrm{dl})$, quadro proteico normale, protidosintesi conservata, marcatori tumorali negativi, VES = $130 \mathrm{sec}$, procalcitonina nei limiti di norma.

Sono risultate negative le sierologie per il virus di Epstein-Barr, Toxoplasma, Citomegalovirus, HIV, Brucella, TBC, Leishmania e Rosolia.

La TC del collo con mdc confermava la natura linfonodale della tumefazione sottomandibolare destra, all'interno della quale si osservavano multiple aree colliquate (Figura 1).

L'esame esteso al torace e all'addome rivelava la presenza di multiple linfoadenopatie in sede mediastinica, ilare epatica, lomboaortica, interportocavale e mesenterica. Il fegato e la milza presentavano aspetto disomogeneo per presenza di numerose aree nodulari ipodense del diametro massimo di $10 \mathrm{~mm}$, di incerta interpretazione (Figura 2).

Inoltre, in stretta continuità con la testa del pancreas, veniva descritta una formazione ovalare solida di $21 \mathrm{~mm} \times 28 \mathrm{~mm}$, anch'essa di dubbia natura (Figura 3 ).

L'iter diagnostico procedeva a questo punto con biopsia chirurgica del pacchetto linfonodale sottomandibolare: l'esame istologico

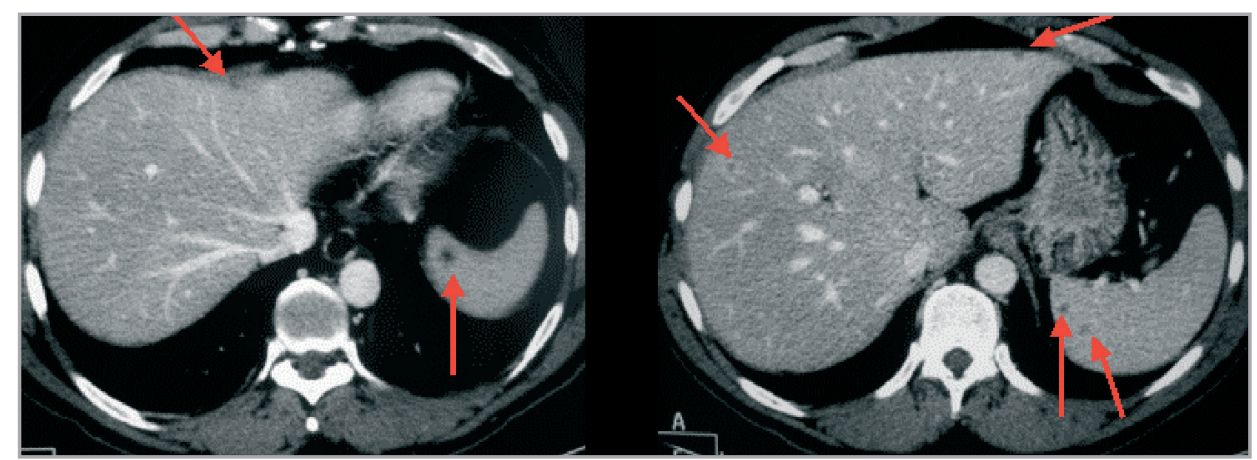




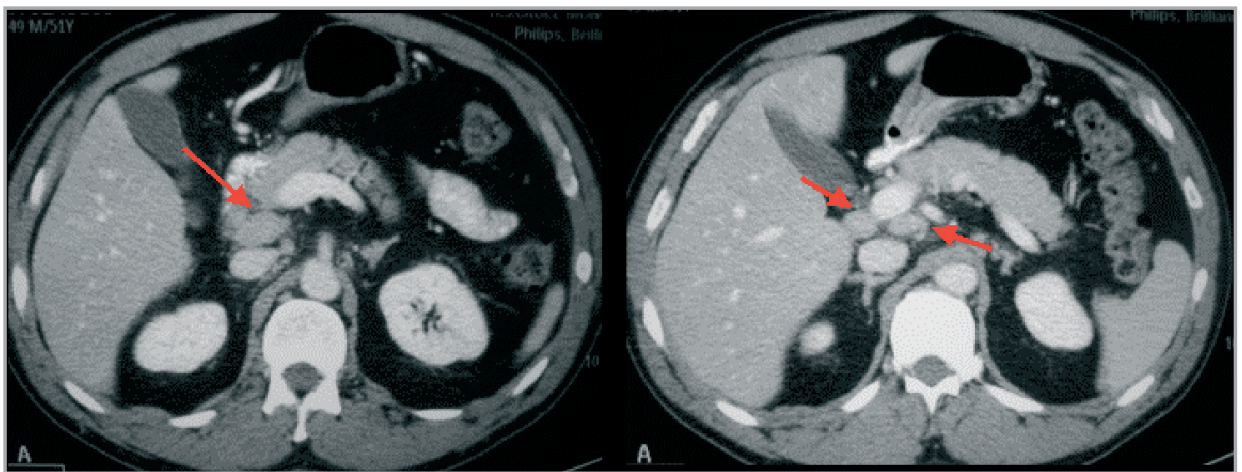

Figura 3

TC addome che fa rilevare una formazione ovalare solida in stretta continuità con la testa del pancreas (frecce)

descriveva a livello macroscopico un quadro di linfadenite granulomatosa necrotizzante con infiltrazione granulocitaria, altamente suggestiva per malattia da graffio di gatto (CSD). La diagnosi veniva poco dopo confermata dal riscontro di franca positività per IgM anti-Bartonella.

L'ecocardiogramma escludeva vegetazioni endocarditiche valvolari.

Nuovamente interrogato per ulteriore ragguaglio anamnestico, il paziente negava ogni contatto con qualsiasi animale domestico o selvatico, ma ricordava la comparsa di una lesione pustolosa della guancia destra, da lui attribuita a foruncolo, circa una settimana prima della comparsa dei sintomi, a risoluzione spontanea completa.

Poiché durante il ricovero le condizioni erano ulteriormente scadute e poiché la febbre persistente e la mancata discesa dei parametri di infiammazioni dichiaravano inefficaci sia la seconda linea di terapia antibiotica (claritromicina $500 \mathrm{mg} /$ bid per 5 giorni) sia la terza (ceftriaxone ev $2 \mathrm{~g} / \mathrm{die}$ per 6 giorni), alla luce della diagnosi acquisita si decideva di trattare il paziente con teicoplanina $600 \mathrm{mg} / \mathrm{die}$ ev associata a ertapenem ev $1 \mathrm{~g} /$ die. Il trattamento, protratto per 3 settimane in regime di Day Hospital, portava a un notevole miglioramento delle condizioni generali, con graduale riduzione delle adenopatie periferiche fino a completa scomparsa. Anche i parametri laboratoristici si sono via via normalizzati e il paziente ha ripreso le normali attività quotidiane. Il secondo dosaggio degli anticorpi antiBartonella eseguito a 3 mesi ha dimostrato completa sieroconversione.

\section{DISCUSSIONE}

Il signor CP era affetto da una patologia di per sé benigna, a risoluzione spontanea e di facile diagnosi nell'ospite immunocom-
Quali sono le peculiarità di questo caso?

Esse consistono:

- nella dimostrazione di una infrequente modalità di contagio;

- in una presentazione di malattia decisamente atipica per un soggetto sano;

- nella scelta di uno schema terapeutico non in linea con le raccomandazioni della letteratura, ma rivelatosi vincente.

petente [1]. Tuttavia il percorso clinico nel nostro caso è stato notevolmente complicato (e rallentato) dalla completa assenza di dati anamnestici.

\section{II patogeno}

Bartonella henselae $(\mathrm{BH})$, identificata nei primi anni '90 e denominata inizialmente Rochalimaea henselae [2], è responsabile del 95\% dei casi di malattia da graffio di gatto (CSD) nell'uomo $[3,4]$.

Alcuni punti nella patogenesi di questa malattia restano da chiarire, ma è ben noto come il gatto sia il più frequente serbatoio naturale di BH, bacillo Gram negativo che si moltiplica negli eritrociti dell'animale [5]. La maggior parte delle evidenze correla quindi la CSD all'esposizione a graffio o morso di gatto, a sua volta infettato da una pulce denominata Ctenocephalides felis, che gioca un ruolo chiave anche nella trasmissione della $\mathrm{BH}$ da gatto a gatto [6].

Meno frequente è la possibilità di infezione diretta dalla pulce all'uomo, mentre non sono note modalità alternative di contagio (es. graffio con oggetti contaminati) [7]; è ragionevole interpretare la lesione similpustolosa descritta in seconda battuta dal nostro paziente, e non più visibile al momento della prima visita, come esito di contatto con il parassita ematofago [8,9].

Il periodo dell'anno in cui il signor CP si è ammalato è in accordo con la letteratura 
che indica nell'autunno e nell'inverno le stagioni di picco massimo di malattia, la cui prevalenza e incidenza in Italia e nel mondo (a parte il Nord America, area in assoluto di maggiore incidenza) è di 9-10 casi/ 100.000 all'anno [10].

\section{La malattia}

La fascia di età a cui appartiene il nostro paziente non è la più frequentemente colpita da CSD: infatti la maggior parte dei soggetti immunocompetenti affetti ha un'età inferiore a 20 anni, sebbene uno studio israeliano nel 2005 abbia dimostrato un'incidenza di circa il 6\% tra persone sane ultrasessantenni [11].

È ben noto come la presentazione clinica di malattia sia molto diversa in base allo stato immunitario dell'ospite. La manifestazione classica nell'immunocompetente prevede, 3-10 giorni dopo il graffio, la comparsa di una lesione cutanea pustolosa, papulosa o vescicolosa, che può persistere per giorni o settimane, guarendo senza lasciare cicatrici. Come già detto, nel nostro caso viene descritta una formazione cutanea con tali caratteristiche ma non ascrivibile a graffio. Patognomonica di CSD è la linfoadenopatia singola locoregionale prossimale al sito di inoculo del patogeno, che compare di solito entro due settimane dal contatto con l'animale. I linfonodi interessati sono localizzati prevalentemente nel collo, incavo ascellare e arti inferiori, in quanto sedi più comuni di graffio o morso; hanno generalmente un diametro di $1-5 \mathrm{~cm}$ e appaiono arrossati e dolenti. In una bassa percentuale di casi (1015\%) si osserva l'evoluzione suppurativa dei linfonodi, mentre la maggior parte di essi regredisce spontaneamente entro 2-6 mesi [12]. Frequenti sintomi aggiuntivi sono costituiti da febbricola, malessere, cefalea, anoressia, faringodinia e poliartralgie.

Il nostro paziente, nonostante la storia anmnestica muta e il buon stato globale di salute, rientrava nel 5-14\% di casi di affetti da CSD severa, a interessamento multiorganico. In queste forme atipiche, in genere osservabili in soggetti defedati, gli organi più frequentemente coinvolti sono fegato, milza, occhio, cuore, osso e SNC [13-17]. Patognomonico della CSD severa è il riscontro radiologico di lesioni ipodense a carico di fegato e milza riferibili a granulomi necrotizzanti (Figure 2 e 3). La funzione epatica è in genere ben conservata, mentre tipica è l'elevazione degli indici di infiammazione sistemica.

\section{La diagnosi}

A quali ipotesi diagnostiche è necessario pensare in un caso come questo?

La CSD si pone in diagnosi differenziale con altre numerose patologie, riassunte nella Tabella I.

Per stabilire la diagnosi di CSD vengono proposti alcuni criteri: ne sono necessari 3 su 4 per identificare la malattia [18]:

- dato anamnestico di contatto con animale (indipendentemente dalla presenza di una lesione cutanea da inoculazione);

- sierologia negativa per altri patogeni comunemente responsabili di adenopatie;

- sierologia positiva per $\mathrm{BH}$ con metodo immunoenzimatico (EIA) o indiretto a fluorescenza (IFA);

- reperto istologico di flogosi granulomatosa necrotizzante coerente con CSD.

\section{La terapia}

I dati sull'utilizzo di antibiotici nel trattamento della CSD derivano principalmente da case report o da piccoli trial, pertanto non esistono linee guida univoche che indichino uno schema di trattamento ottimale.

Uno studio retrospettivo condotto nei primi anni ' 90 ha dimostrato l'efficacia di rifampicina, ciprofloxacina, trimetoprim/ sulfametossazolo e gentamicina in termini di riduzione di giorni di malattia nei soggetti trattati rispetto ai non trattati [19].

Un altro trial prospettico randomizzato controllato condotto su 29 pazienti immunocompetenti (bambini e adulti) con presentazione tipica di malattia ha invece testato l'efficacia di azitromicina in termini di riduzione di volume delle adenopatie periferiche. Non è stata dimostrata alcuna efficacia di questo antibiotico nel trattamento di forme complicate di CSD, né per prevenirne l'evoluzione da forma semplice a disseminata [20].

Le raccomandazioni della letteratura più recente in materia di trattamento possono essere così riassunte:

- nelle presentazioni tipiche di CSD sia nel bambino sia nell'adulto non si ritiene indicato il trattamento antibiotico;

- in caso di linfoadenopatia estesa può essere considerato l'utilizzo di azitromicina:

- nell'adulto: $500 \mathrm{mg}$ per os il primo giorno, seguito da $250 \mathrm{mg}$ per os dai giorni 2 a 5 in dose singola giornaliera; 


\begin{tabular}{|c|c|c|}
\hline \multirow[t]{4}{*}{ Malattie infettive } & Batteriche & $\begin{array}{l}\text { Streptococchi, stafilococchi, malattia da graffio } \\
\text { di gatto, brucellosi, tularemia, peste, tubercolosi, } \\
\text { sifilide, difterite, lebbra }\end{array}$ \\
\hline & Virali & $\begin{array}{l}\text { Virus di Epstein-Barr, Citomegalovirus, Herpes, } \\
\text { Rosolia, Morbillo, Adenovirus, HIV }\end{array}$ \\
\hline & Fungine & $\begin{array}{l}\text { Istoplasmosi, coccidiodomicosi, } \\
\text { paracoccidiodomicosi }\end{array}$ \\
\hline & Parassitarie & Toxoplasmosi, leishmaniosi, tripanosomiasi, filariosi \\
\hline Malattie immunologiche & $\begin{array}{l}\text { Artrite reumatoide, connettivite mista, lupus } \\
\text { eritematoso sistemico, sindrome di Sjögren, } \\
\text { malattia da siero, ipersensibilità da farmaci, Graft } \\
\text { Versus Host Disease }\end{array}$ & \\
\hline \multirow[t]{2}{*}{ Malattie maligne } & Ematologiche & $\begin{array}{l}\text { Linfoma di Hodgkin, linfoma non-Hodgkin, leuce- } \\
\text { mia linfoblastica acuta, leucemia linfatica cronica, } \\
\text { istiocitosi maligna, amiloidosi }\end{array}$ \\
\hline & Metastatiche & Da numerose sedi primitive \\
\hline Malattie da accumulo di lipidi & Gaucher, Niemann-Pick, Fabry, Tangier & \\
\hline Altro & $\begin{array}{l}\text { Malattia di Castelman, sarcoidosi, granulomatosi } \\
\text { linfomatoide, malattia di Kawasaki, istiocitosi X, } \\
\text { febbre mediterranea familiare, pseudotumore in- } \\
\text { fiammatorio del linfonodo }\end{array}$ & \\
\hline
\end{tabular}

- nel bambino: $10 \mathrm{mg} / \mathrm{kg}$ per os il primo giorno, seguito da $5 \mathrm{mg} / \mathrm{kg}$ nei giorni 2-5 (raccomandazione BI) [4];

- nei pazienti intolleranti all'azitromicina regimi alternativi comunemente usati sono:

- claritromicina $(15-20 \mathrm{mg} / \mathrm{kg}$ divisi in 2 dosi se peso $<45,5 \mathrm{~kg}$, o $500 \mathrm{mg} / \mathrm{bid}$ per pazienti di peso > 45,5 kg);

- rifampicina (bambini: $10 \mathrm{mg} / \mathrm{kg}$ bid; adulti: $300 \mathrm{mg} / \mathrm{bid}$ );

- trimetoprim-sulfametossazolo (nei bambini: trimetoprim $8 \mathrm{mg} / \mathrm{kg}$ al giorno + sulfametossazolo $40 \mathrm{mg} / \mathrm{kg}$ al giorno, suddiviso in due dosi; adulti: una compressa bid);

- ciprofloxacina (per i pazienti > 17 anni di età, $500 \mathrm{mg} / \mathrm{bid}$ );

- nelle forme complicate sia epatospleniche sia neurologiche i pochi dati disponibili in letteratura propendono per utilizzare sempre antibiotici. In particolare nell'adulto:

- doxiciclina $100 \mathrm{mg} / \mathrm{bid}$ per os per 4-6 settimane;

- rifampicina $300 \mathrm{mg} / \mathrm{bid}$ per os per 4-6 settimane [21].

Anche un piccolo trial condotto su 19 bambini con manifestazioni epatospleniche ha dimostrato l'efficacia di rifampicina al dosaggio di $20 \mathrm{mg} / \mathrm{kg}$ al giorno per 14 giorni (somministrata da sola o in combinazione con gentamicina o trimetoprim-sulfametossazolo) [22].

La durata ottimale del trattamento non è del tutto chiara ma la maggior parte degli studi la indica non inferiore a 14 giorni nelle forme epatospleniche e 4-6 settimane in caso di coinvolgimento neurologico.

Lo schema di terapia da noi utilizzato, un'associazione di ertapenem e teicoplanina, si presta senz'altro a critiche, prima fra tutte l'impossibilità di confronto con la letteratura in termini di efficacia. Ricordiamo che il paziente, allergico ai chinolonici, era stato precedentemente trattato con alcune tra le classi antibiotiche più spesso raccomandate (amoxicillina/acido clavulanico, claritromicina, ceftriaxone) senza alcun risultato apprezzabile. Il problema urgente di aggredire lesioni intra-addominali necrotizzanti ha indirizzato la scelta del carbapenemico. L'aggiunta, probabilmente ridondante, di teicoplanina (antibiotico a spettro d'azione prevalente su batteri Gram positivi) ha comunque garantito una più ampia copertura antibatterica in un paziente a questo punto defedato e persistentemente febbrile.

\section{DISCLOSURE}

Gli Autori dichiarano di non avere conflitti di interesse di natura finanziaria in merito ai temi trattati nel presente articolo.

\section{Tabella I}

Diagnosi differenziali per la malattia da graffio di gatto 


\section{BIBLIOGRAFIA}

1. Bass JW, Vincent JM, Person DA. The expanding spectrum of Bartonella infections: cat-scratch disease. Pediatr Infect Dis J 1997; 16: 163-79

2. Wear DJ, Margileth AM, Hadfield TL, Fischer GW. Cat scratch disease: a bacterial infection. Science 1983; 221: 1403-5

3. Regnery RL, Olson JG, Perkins BA, Bibb W. Serological response to "Rochalimaea henselae" antigen in suspected cat-scratch disease. Lancet 1992; 339: 1443-5

4. Rolain JM, Brouqui P, Koehler JE, Maguina C, Dolan MJ, Raoult D. Recommendations for treatment of human infections caused by Bartonella species. Antimicrob Agents Chemother 2004; 48: 1921-33

5. Jacomo V, Kelly PJ, Raoult D. Natural history of Bartonella infections (an exception to Koch's postulate). Clin Diagn Lab Immunol 2002; 9: 8-18

6. Dehio C. Molecular and cellular basis of Bartonella pathogenesis. Annu Rev Microbiol 2004; 58: 365-90

7. Mosbacher M, Elliott SP, Shehab Z, Pinnas JL, Klotz JH, Klotz SA. Cat scratch disease and arthropod vectors: more to it than a scratch? J Am Board Fam Med 2010; 23: 685-6

8. Koehler JE, Glaser CA, Tappero JW. Rochalimaea henselae infection. A new zoonosis with the domestic cat as reservoir. JAMA 1994; 271: 531-5

9. Chomel BB. Experimental transmission of Bartonella henselae by the cat flea.J Clin Microbiol 1996; 34: 1952-6

10. Windsor JJ. Cat-scratch disease: epidemiology, aetiology and treatment. Br J Biomed Sci 2001; 58: $101-10$

11. Ben-Ami R, Ephros M, Avidor B, Katchman E, Varon M, Leibowitz C et al. Cat-scratch disease in elderly patients. Clin Infect Dis 2005; 41: 969-74

12. Ridder GJ, Boedeker CC, Technau-Ihling K, Sander A. Cat-scratch disease: otolaryngologic manifestations and management. Otolaryngol Head Neck Surg 2005; 132: 353-8

13. Curi AL, Machado D, Heringer G, Campos WR, Lamas C, Rozental T. Cat-scratch disease: ocular manifestations and visual outcome. Int Ophthalmol 2010; 30: 553-8

14. Suhler EB, Lauer AK, Rosenbaum JT. Prevalence of serologic evidence of cat scratch disease in patients with neuroretinitis. Ophthalmology 2000; 107: 871-6

15. Baylor P, Garoufi A, Karpathios T, Lutz J, MogelofJ, Moseley D. Transverse myelitis in 2 patients with Bartonella henselae infection (cat scratch disease). Clin Infect Dis 2007; 45: e42-e45

16. Maman E, Bickels J, Ephros M, Paran D, Comaneshter D, Metzkor-Cotter E. Musculoskeletal manifestations of cat scratch disease. Clin Infect Dis 2007; 45: 1535-40

17. Hajjaji N, Hocqueloux L, Kerdraon R, Bret L. Bone infection in cat-scratch disease: a review of the literature. J Infect 2007; 54: 417-21

18. Margileth AM. Recent advances in diagnosis and treatment of cat scratch disease. Curr Infect Dis Rep 2000; 2: 141-6

19. Margileth AM. Antibiotic therapy for cat-scratch disease: clinical study of therapeutic outcome in 268 patients and a review of the literature. Pediatr Infect Dis J 1992; 11: 474-8

20. Bass JW, Freitas BC, Freitas AD, Sisler CL, Chan DS, Vincent JM. Prospective randomized double blind placebo-controlled evaluation of azithromycin for treatment of cat-scratch disease. Pediatr Infect Dis J 1998; 17: 447-52

21. Reed JB, Scales DK, Wong MT, Lattuada CP Jr. Bartonella henselae neuroretinitis in cat scratch disease. Diagnosis, management, and sequelae. Ophthalmology 1998; 105: 459-66

22. Arisoy ES, Correa AG, Wagner ML, Kaplan SL. Hepatosplenic cat-scratch disease in children: selected clinical features and treatment. Clin Infect Dis 1999; 28: 778-84 